\title{
Alteraciones sistémicas asociadas a Hipomineralización Molar Incisivo (HMI). Una revisión de literatura
}

\author{
Nely Arely García de Batres, ${ }^{1}$ (D) Magdalena Raquel Torres Reyes, ${ }^{2}$ \\ Geovanni Arturo Ramírez Martínez, ${ }^{3}$ (D) Guillermo Alfonso Aguirre Escobar. ${ }^{4}$
}

\begin{abstract}
Resumen: El estudio de hipomineralización en molares e incisivos fue descrito por primera vez en el año 1970, posteriormente en 2001 el término Hipomineralización Molar Incisivo (HMI) fue sugerido por la Academia Europea de Odontología Pediátrica (EAPD) para referirse a este como un defecto específico del desarrollo del esmalte; ya para el año 2003 se estandarizaron los criterios de diagnóstico clínico. La HMI es un defecto del esmalte dentario ampliamente estudiado, sin embargo, hasta la fecha los factores de riesgo no son concluyentes, pero se considera de origen sistémico y multifactorial. Las implicaciones sistémicas pueden acontecer en periodos específicos (pre, peri y posnatal) considerados periodos importantes y críticos en el desarrollo de la vida humana. Objetivo: aportar la mejor evidencia científica disponible sobre los factores sistémicos asociados a la Hipomineralización Molar Incisivo. Materiales y métodos: Se realizó una búsqueda sistematizada seleccionando estudios primarios en bases de datos electrónicas: Pub Med, biblioteca Cochrane e Hinari a través de la pregunta PICO. Resultados: 115 estudios fueron identificados a través de la búsqueda electrónica de estos solo 18 fueron elegibles según los criterios de inclusión. Conclusiones: Son múltiples los posibles factores sistémicos asociados con HMI, entre ellos resaltan complicaciones en el embarazo como: fiebres altas, hipertensión arterial, diabetes gestacional, procesos infecciosos, uso frecuente de antibióticos y desnutrición, así como parto prematuro, bajo peso al nacer y las enfermedades respiratorias, fiebre y otitis en los primeros años de vida del niño.
\end{abstract}

Palabras clave: Etiología, Esmalte Dental, Exposición Materna, Odontología pediátrica (Odontopediatria), Amelogénesis.

\section{Alterações sistêmicas associadas à Hipomineralização Molar Incisiva (HMI). Uma revisão da literatura.}

\begin{abstract}
Resumo: O estudo da Hipomineralização nos molares e incisivos foi descrito pela primeira vez no ano de 1970 , posteriormente no 2001 o termo Hipomineralização Molar Incisivo (HMI) foi sugerido pela Academia Europeia de Odontopediatria (EAPD) para se referir a este como um defeito específico do desenvolvimento do esmalte, já no ano 2003 se padronizaram os critérios de diagnóstico clínico. A HMI é um defeito do esmalte dentário amplamente estudado, no entanto até hoje os fatores de risco ainda não estão totalmente esclarecidos, mas se considera como de origem sistémico e multifatorial. As implicações sistémicas podem acontecer em períodos específicos (pre, peri e pós natal) que se consideram períodos importantes e críticos no desenvolvimento da vida humana. Objetivo: aportar a melhor evidencia científica disponível sobre os fatores sistémicos associados à Hipomineralização Molar Incisivo. Materiais e métodos: foi realizada uma busqueda sistematizada selecionando estudos primários em bases de dados eletrônicas: Pub Med, biblioteca Cochrane e Hinari a través da pergunta PICO. Resultados: 115 estudos foram identificados a través da busqueda eletrônica dos quais unicamente 18 foram elegíveis segundo os critérios de inclusão. Conclusões: São muitos os possíveis fatores sistémicos associados com HMI entre eles destacam complicações na gravidez como: febres altas, hipertensão arterial,

\footnotetext{
1 Residente de la Maestría de Odontopediatria, Facultad de Odontología, Universidad de El Salvador.

2 Mestre en Odontología. Especialista en Odontopediatria con énfasis en Odontología del bebé. Profesora de Posgrado de la Maestría de Odontopediatria, Facultad de Odontología, Universidad de El Salvador.

3 Especialista en Odontopediatría con énfasis en Odontología del bebé. Profesor posgrado de la Maestría Odontopediatría, Facultad de Odontología, Universidad de El Salvador.

4 Especialista en Odontología Preventiva, Maestría en Epidemiologia y Salud Pública, Profesor e investigador, Facultad de Odontología, Universidad de El Salvador.
} 
diabetes gestacional, processos infecciosos, uso frequente de antibióticos e desnutrição, assim como parto prematuro, baixo peso ao nascer e as doenças respiratórias, febre e otitis nos primeiros anos de vida da criança.

Palabra-chave: Etiologia, Esmalte dentário, Exposição Materna, Odontopediatria, Amelogênese.

\section{Systemic alterations associated with Incisor Molar Hypomineralization (HMI). A literature review}

Abstract: The study of Hypomineralization in molars and incisors was described for the first time in the year 1970. Later in 2001, the term Molar Incisor Hypomineralization (MIH) was suggested by the European Academy of Paediatric Dentistry (EAPD) to refer to this as a specific enamel development defect, and in 2003, the clinical diagnostic criteria were standardized. MIH is a widely studied tooth enamel defect; however, to date, the risk factors are not conclusive but, it is considered of systemic and multifactorial origin. The systemic implications can occur in specific periods (pre, peri, and postnatal) considered important and critical periods of the development of human life. Objective: to provide the best available scientific evidence on the systemic factors associated with molar incisor Hypomineralization. Methodology: A systematized search was varied out selecting primary studies in electronic database: Pub Med, Cochrane and Hinari library, through the PICO question. Results: 115 studied were identified through the electronic search. Of these, only 18 were found eligible according to the inclusion criteria. Conclusions: There are multiple possible systemic factors associated with $\mathrm{MIH}$. Among them, certain complications in pregnancy stand out, such as: Hugh fevers, arterial hypertension, gestational diabetes, infectious processes, frequent use of antibiotics and malnutrition, as well as premature delivery, low birth weight, and respiratory diseases, fever and otitis in the first years of the child's life.

Key words: Etiology, Dental Enamel, Maternal Exposure, Pediatric dentistry, Amelogenesis.

\section{Introducción}

El esmalte dental es un tejido único, altamente mineralizado $y$ de origen ectodérmico, que una vez formado, se caracteriza por la ausencia de actividad metabólica. Ello determina que, sí se presentan disturbios durante su desarrollo, se pueden manifestar como defectos permanentes en los dientes cuando estos erupcionan. ${ }^{1}$ La amelogénesis se ha dividido en tres etapas principales del ciclo de vida de los ameloblastos: secreción, transición y maduración. ${ }^{2}$ En la etapa secretora se produce la matriz del esmalte, en la etapa de transición existe una transformación de los ameloblastos secretores la cual se acompaña de una mineralización masiva y en la etapa de maduración los ameloblastos ya maduros regulan la mineralización final del esmalte. ${ }^{2}$ En términos histológicos, HMI resulta de una alteración en la acción de los ameloblastos durante la fase de maduración que persiste aproximadamente hasta los 3 años para los primeros molares e incisivos permanentes. ${ }^{3}$

En general, los factores sistémicos que perturban los ameloblastos durante la etapa secretora causan restricción del alargamiento de los cristales y dan como resultado un esmalte patológicamente delgado o hipoplásico. Las alteraciones durante la etapa de transición y maduración de la amelogénesis dan como resultado un esmalte patológicamente blando (hipomadurado, hipomineralizado) de espesor normal. ${ }^{2}$ La mineralización 
del esmalte comienza justo antes del nacimiento, incluso los primeros molares permanentes comienza a desarrollarse durante el cuarto mes de gestación. ${ }^{4}$ La formación del esmalte en su conjunto lleva aproximadamente mil días. ${ }^{5}$ Aunque la amelogénesis está controlada estrictamente por la genética esta es sensible a las perturbaciones ambientales, lo que resulta en diferentes tipos de defectos. $^{6}$

El término Hipomineralización molar incisivo se describe por primera vez en Suecia en 1970,7 el término HMI fue sugerido en 2001 y luego estandarizado por la Academia Europea de Odontología Pediátrica (EAPD) por medio del establecimiento de criterios diagnósticos en $2003,{ }^{8} \mathrm{HMI}$ es un defecto cualitativo en el desarrollo del esmalte de posible origen sistémico y multifactorial que afecta de uno a cuatro de los primeros molares permanentes pudiendo o no comprometer los incisivos permanentes. ${ }^{9,10}$ Inicialmente estos defectos del desarrollo del esmalte se registraron afectando el grupo de molares e incisivos permanentes, sin embargo, recientes investigaciones realizadas por Elfrink et al. en 2015, ${ }^{11}$ observaron defectos similares en segundos molares y ocasionalmente en caninos deciduos siendo un fuerte predictor para la aparición de hipomineralizaciones en la dentición permanente. ${ }^{12-15}$ Este tipo de hipomineralización en la dentición decidua se define como Hipomineralización de Segundo Molar Deciduo (HSMD) que se caracteriza por afectar de 1 a 4 de los segundos molares deciduos. ${ }^{12,16}$

Tanto la HMI como la HSMD, se consideran defectos cualitativos del esmalte que se manifiestan en opacidades blancas, amarillas o marrones, según el grado de afectación. ${ }^{17}$ Este tipo de defecto es cada vez más frecuente en la práctica odontológica y representa un gran desafío para el clínico dental, especialmente el odontopediatra.

Existen diversas revisiones de literatura sobre Hipomineralización en dentición permanente y decidua mostrando una alta prevalencia de este defecto del esmalte a nivel mundial. ${ }^{11,18,19}$ No existe un consenso claro sobre la prevalencia de HMI, esta es ampliamente variada entre los diferentes estudios, eso debido a la falta de estandarización de los criterios clínicos y de evaluación utilizados. ${ }^{20}$ Una reciente revisión de literatura en relación a prevalencia mostró una amplia variación tanto para HMI como para HSMD (HMI 2.9-44\%; HSMD 0-21.8\%). ${ }^{11}$ Sin embargo, estudios indican que es posible que se esté produciendo un incremento. ${ }^{20-21}$

Actualmente la $\mathrm{HMI}$ es el defecto del esmalte más frecuente presentando una alta prevalencia a nivel mundial, ${ }^{22}$ convirtiéndose así en un problema de salud pública.

La etiología de HMI todavía no se comprende completamente, los posibles factores de riesgo no son concluyentes y están poco definidos; aún no existe una unificación por parte de los investigadores con respecto a este punto, sin embargo, se describen factores de riesgo ambientales, genéticos, ${ }^{9}$ así como también, implicaciones sistémicas, es decir, eventos médicos generales que pueden afectar la salud de la gestante o del niño en sus primeros años de vida, implicando en los períodos prenatal, perinatal y postnatal, etapas 
consideradas como importantes y críticas en el desarrollo de la vida humana. . $^{1,7,7,20,22}$

Los rangos de edades para cada uno de estos periodos son los siguientes:

Período prenatal (de 0 a 36-38 semanas en útero)

Período perinatal (desde el nacimiento hasta los 28 días posteriores al nacimiento)

Período postnatal (de 29 días a 4 años). ${ }^{21}$

Por tal razón desde el tercer trimestre del embarazo hasta los 3 años de edad, se considera una etapa sensible de desarrollo del niño en el cual las coronas de los primeros molares e incisivos permanentes se mineralizan. ${ }^{2}$

Entre las implicaciones sistémicas asociadas a la presencia de $\mathrm{HMI}$ podemos destacar:

Complicaciones o enfermedades durante el embarazo, entre las más reportadas se encuentra la diabetes gestacional, deficiencias vitamínicas y dificultades respiratorias.

Al momento del parto se han asociado con parto prematuro, el tipo de parto y el tiempo, si este fue prolongado hay mayor riesgo que el niño desarrolle HMI. Posterior al parto, las alteraciones más frecuentes reportadas por la evidencia científica son principalmente problemas respiratorios como neumonía y asma bronquial, así como amigdalitis, adenoiditis, fiebres, otitis media y la ingesta de antibióticos. ${ }^{21,24-40}$
El objetivo de esta revisión de literatura es aportar la mejor evidencia científica disponible sobre las implicaciones sistémicas asociadas a Hipomineralización Molar Incisivo.

Es esencial conocer y comprender de una manera clara sobre la etiología de esta condición; principalmente para la identificación temprana y manejo oportuno de los grupos de riesgo potenciales para desarrollar HMI. Realizar una buena anamnesis permitirá la identificación de factores de riesgo para establecer planes de tratamiento oportunos orientados a la prevención y educación en salud, así como para monitorear las posibles secuelas que los dientes afectados por $\mathrm{HMI}$ pueden presentar debido a su vulnerabilidad: fracturas pos eruptivas, lesiones de caries y sensibilidad dentaria que afectan la calidad de vida del niño.

\section{Materiales y métodos}

Fuentes de información y estrategia de búsqueda

Se realizó una búsqueda sistemática utilizando las bases de datos electrónicas Medline (PubMed), Embase, Cochrane Library y revistas científicas especializadas como Caries Research, American Academy of Pediatric Dentistry, International Journal of Paediatric Dentistry, European Academy of Paediatric Dentistry, European Archives of Paediatric Dentistry, Original Research of Pediatric Dentistry y Community Dentistry and Oral Epidemiology, para investigar el vínculo entre $\mathrm{HMI}$ y factores sistémicos prenatales, perinatales, y posnatales.

Se determinó una estrategia de búsqueda. Los términos se obtuvieron con la ayuda 
de la biblioteca virtual en salud, a través de los descriptores DeCs, uso de vocabulario controlado (términos $\mathrm{MeSH}$ ) de la base de datos PubMed. Estas palabras se combinaron con el operador booleano OR dentro de cada concepto de la estrategia de búsqueda. Los conceptos de la pregunta PICO (Participante, Intervención, comparación, resultados y diseño del estudio) se combinaron luego con el operador booleano AND. La combinación de las siguientes palabras fue utilizada para la búsqueda de estudios relevantes: (Molar Incisor Hypomineralization) AND (aetiology) ((molar-incisor) AND (Hypomineralization)) AND (aetiology), ((pregnancy) AND (molarincisor)) OR (Hypomineralization), (Perinatal Care) AND (Hypomineralization molarincisor), (Dental Enamel Hypoplasia) AND (pathogenesis). (Aetiology) OR (molar-incisorhypomineralization)

Se describe la estrategia PICO del presente estudio:

P Población infantil y mujeres embarazadas.

I Factores sistémicos prenatales, perinatales y postnatales.

C Ausencia de factores sistémicos.

O Relación factores sistémicos a Hipomineralización Molar Incisivo.

Pregunta: ¿Existe asociación entre los factores sistémicos prenatales, perinatales y postnatales con la Hipomineralización Molar Incisivo?
Proceso de recolección de datos

Se realizó la búsqueda de estudios primarios (estudios de cohorte específicos, estudios de casos y controles, poblacionales, epidemiológicos y estudios transversales con muestras poblacionales de gran tamaño) para extraer artículos con énfasis en las variables de estudio consideradas (factores sistémicos prenatales, perinatales y posnatales asociados con $\mathrm{HMI}$ ) lo que permitió realizar una primera selección, revisión y posterior exclusión de artículos.

Todos los estudios originales involucrando niños de todas las edades que reportaron resultados de HMI y factores sistémicos asociados, fueron considerados elegibles para esta revisión. La búsqueda fue limitada a estudios humanos e incluyó solo publicaciones en idioma inglés. Fueron excluidas las publicaciones duplicadas, informes de casos y artículos en otro idioma que no fuese inglés.

La búsqueda de artículos publicados se limitó al período comprendido entre enero de 2010 a diciembre de 2019.

Los estudios seleccionados e incluidos en esta revisión se presentaron de forma resumida en tablas, donde se muestran las principales características de los estudios seleccionados y sus conclusiones, así como un resumen de los estudios seleccionados presentando sus resultados según los periodos del desarrollo afectados por los factores sistémicos estudiados.

Los datos considerados en tabla 1 fueron: autor, año, país, diseño de estudio, rango de edad, tamaño de muestra, reclutamiento de pacientes, instrumentos de colecta de 
Tabla 1: Resumen de las características de los estudios seleccionados y sus conclusiones.

\begin{tabular}{|c|c|c|c|c|c|c|c|c|c|c|}
\hline \multirow{2}{*}{ Autor } & \multirow{2}{*}{ Año } & \multirow{2}{*}{ País } & \multirow{2}{*}{$\begin{array}{l}\text { Diseño de } \\
\text { estudio }\end{array}$} & \multirow{2}{*}{$\begin{array}{l}\text { Rango de } \\
\text { edad }\end{array}$} & \multirow{2}{*}{$\begin{array}{l}\text { Tamaño de } \\
\text { muestra }\end{array}$} & \multirow{2}{*}{$\begin{array}{l}\text { Reclutamiento de } \\
\text { pacientes }\end{array}$} & \multicolumn{2}{|c|}{$\begin{array}{l}\text { Instrumentos de } \\
\text { colecta de datos }\end{array}$} & \multirow{2}{*}{ Conclusiones } & \multirow{2}{*}{ Valor $\mathrm{p}$} \\
\hline & & & & & & & $\begin{array}{l}\text { Examen } \\
\text { clínico }\end{array}$ & Cuestionario & & \\
\hline $\begin{array}{l}\text { Souza JF, } \\
\text { Costa-Silva } \\
\text { CM, Jeremias } \\
\text { F, San- } \\
\text { tos-Pinto L, } \\
\text { Zuanon ACC, } \\
\text { Cordeiro } \\
\text { RCL. }{ }^{33}\end{array}$ & 2012 & Brasil & $\begin{array}{l}\text { Estudio } \\
\text { transversal }\end{array}$ & 6 a 12 años & 903 niños & $\begin{array}{l}\text { Residentes en } \\
\text { zonas rurales y } \\
\text { urbanas de la } \\
\text { ciudad de Botelhos, } \\
\text { estado de Minas } \\
\text { Gerais. }\end{array}$ & $\begin{array}{l}\text { Según los } \\
\text { criterios de la } \\
\text { EAPD. }\end{array}$ & $\begin{array}{l}\text { Cuestionario } \\
\text { estructurado } \\
\text { de historia } \\
\text { clínica, desde } \\
\text { el embarazo } \\
\text { hasta el } \\
\text { tercer año } \\
\text { de vida del } \\
\text { niño. }\end{array}$ & $\begin{array}{l}\text { Factores prenatales: } \\
\text { problemas de salud } \\
\text { durante el embarazo } \\
\text { como la infección de } \\
\text { garganta, la fiebre } \\
\text { alta y la amoxicilina } \\
\text { asociadas con otros } \\
\text { antibióticos presenta- } \\
\text { ron vínculo entre HMI. }\end{array}$ & $\begin{array}{l}P=0,01 \\
P=0,02 \\
P=0,04\end{array}$ \\
\hline $\begin{array}{l}\text { Sönmez H, } \\
\text { Yildirim G, } \\
\text { Bezgin T. }\end{array}$ & 2012 & Turquía & $\begin{array}{l}\text { Estudio epi- } \\
\text { demiológico }\end{array}$ & $\begin{array}{l}\text { de } 7 \text { a } 12 \\
\text { años }\end{array}$ & $\begin{array}{l}2,029 \text { niñas } \\
2,020 \text { niños }\end{array}$ & $\begin{array}{l}\text { Escuelas primarias } \\
\text { escuelas ubicadas } \\
\text { en las áreas urba- } \\
\text { nas de los cinco } \\
\text { centros municipios } \\
\text { de Ankara, Turquía. }\end{array}$ & $\begin{array}{l}\text { Según los } \\
\text { criterios de la } \\
\text { EAPD. }\end{array}$ & Cuestionario. & $\begin{array}{l}\text { Se encontró que la } \\
\text { HMI estaba asociado } \\
\text { con la prematuridad, } \\
\text { problemas gastroin- } \\
\text { testinales, neumonía, } \\
\text { fiebre frecuente, } \\
\text { sarampión y varicela } \\
\text { antes de los } 4 \text { años. }\end{array}$ & $\begin{array}{l}P=0,035 \\
P=0,05\end{array}$ \\
\hline $\begin{array}{l}\text { Durmus B, } \\
\text { Abbasoglu } \\
\text { Z, Peker S, } \\
\text { Kargul B. }^{35}\end{array}$ & 2013 & Turquía & $\begin{array}{l}\text { Estudio } \\
\text { transversal }\end{array}$ & 7 a 14 años & $\begin{array}{l}104 \text { niñas } \\
124 \text { niños }\end{array}$ & $\begin{array}{l}\text { Facultad de } \\
\text { Odontología de } \\
\text { la Universidad de } \\
\text { Mármara. }\end{array}$ & $\begin{array}{l}\text { Según los } \\
\text { criterios de la } \\
\text { EAPD. }\end{array}$ & Cuestionario. & $\begin{array}{l}\text { Factores prenatales: } \\
\text { Bronquitis durante el } \\
\text { embarazo. } \\
\text { Factores posnatales: } \\
\text { consumo prolongado } \\
\text { de medicamentos } \\
\text { para enfermedades } \\
\text { infantiles como el } \\
\text { asma y la amigdalitis } \\
\text { antes de los tres años } \\
\text { de vida. }\end{array}$ & $\begin{array}{l}P=0,029 \\
P=0,006 \\
P=0,05 \\
P=0,05\end{array}$ \\
\hline $\begin{array}{l}\text { Kühnisch } \\
\text { J, Mach D, } \\
\text { Thiering E, } \\
\text { Brockow I, } \\
\text { Hoffmann U, } \\
\text { Neumann C, } \\
\text { et al } \\
28\end{array}$ & 2014 & Alemania & $\begin{array}{l}\text { Estudio de } \\
\text { cohorte de } \\
\text { nacimiento }\end{array}$ & 10 años & 692 niños & $\begin{array}{l}\text { Clínicas de Obste- } \\
\text { tricia en Munich y } \\
\text { Wesel, Alemania, }\end{array}$ & $\begin{array}{l}\text { Según los } \\
\text { criterios de la } \\
\text { EAPD. }\end{array}$ & $\begin{array}{l}\text { Cuestio- } \\
\text { nario. }\end{array}$ & $\begin{array}{l}\text { Factores posnatales } \\
\text { como: las enfermeda- } \\
\text { des respiratorias antes } \\
\text { de los } 4 \text { años (asma } \\
\text { bronquial, bronquitis, } \\
\text { neumonía) parecen } \\
\text { estar directa o indirec- } \\
\text { tamente relacionadas } \\
\text { con HMI. }\end{array}$ & $P=0,05$ \\
\hline $\begin{array}{l}\text { Ghanim A, } \\
\text { Manton D, } \\
\text { Bailey D, } \\
\text { Mariño R, } \\
\text { Morgan M. }{ }^{21}\end{array}$ & 2013 & Irak & $\begin{array}{l}\text { Estudio } \\
\text { transversal }\end{array}$ & 7 a 9 años & 823 niños & $\begin{array}{l}\text { comunidad iraquí } \\
\text { residente de la } \\
\text { ciudad de Mosul. }\end{array}$ & $\begin{array}{l}\text { Según los } \\
\text { criterios de la } \\
\text { EAPD. }\end{array}$ & $\begin{array}{l}\text { Un cues- } \\
\text { tionario } \\
\text { (modificado } \\
\text { de Whatling } \\
\text { y Fearne). }\end{array}$ & $\begin{array}{l}\text { Factores perinatales } \\
\text { como: hipocalcemia y } \\
\text { dificultad respiratoria. } \\
\text { Posnatales como: neu- } \\
\text { monía, otitis, fiebre } \\
\text { alta, amigdalitis. }\end{array}$ & $\begin{array}{l}P=0,04 \\
P=0,03 \\
P=0,01 \\
P=0,03 \\
P=0,04 \\
P=0,04 \\
P=0,001\end{array}$ \\
\hline $\begin{array}{l}\text { Allazzam } \\
\text { SM, Alaki } \\
\text { SM, Sadek A, } \\
\text { Meligy E }{ }^{36}\end{array}$ & 2014 & $\begin{array}{l}\text { Arabia } \\
\text { Saudita }\end{array}$ & $\begin{array}{l}\text { Estudio } \\
\text { transversal }\end{array}$ & 8 a 12 años & $\begin{array}{l}134 \text { niños } \\
133 \text { niñas }\end{array}$ & $\begin{array}{l}\text { Clínicas dentales } \\
\text { pediátricas de } \\
\text { la Facultad de } \\
\text { Odontología de la } \\
\text { Universidad King } \\
\text { Abdulaziz. }\end{array}$ & $\begin{array}{l}\text { Examen } \\
\text { dental. }\end{array}$ & Cuestionario. & $\begin{array}{l}\text { HMI está significa- } \\
\text { tivamente asociada } \\
\text { con enfermedades } \\
\text { infantiles durante } \\
\text { los primeros } 4 \text { años } \\
\text { de vida, incluyendo } \\
\text { asma, infecciones ade- } \\
\text { noideas, amigdalitis, } \\
\text { fiebre y consumo de } \\
\text { antibióticos. }\end{array}$ & $\begin{array}{l}P=0,001 \\
P=0,001 \\
P=0,001 \\
P=0,014\end{array}$ \\
\hline $\begin{array}{l}\text { Pitiphat W, } \\
\text { Luangchai- } \\
\text { chaweng S, } \\
\text { Pungchan- } \\
\text { chaikul P, } \\
\text { Angwara- } \\
\text { vong O, } \\
\text { Chansamak } \\
\mathrm{N}^{37}\end{array}$ & 2014 & Tailandia & $\begin{array}{l}\text { Estudio } \\
\text { transversal }\end{array}$ & 7 a 8 años & 420 niños & $\begin{array}{l}\text { zonas urbanas } \\
\text { de Khon Kaen, } \\
\text { Tailândia. }\end{array}$ & $\begin{array}{l}\text { Examen } \\
\text { clínico. }\end{array}$ & Cuestionario. & $\begin{array}{l}\text { Afecta período prena- } \\
\text { tal y perinatal: Cesárea } \\
\text { y el parto vaginal } \\
\text { complicado. Período } \\
\text { posnatal: asma y } \\
\text { uso de antibióticos } \\
\text { durante los primeros } \\
3 \text { años de vida son } \\
\text { factores de riesgos } \\
\text { para MIH. }\end{array}$ & $\begin{array}{l}P<0,001 \\
P=0,001 \\
P=0,01\end{array}$ \\
\hline $\begin{array}{l}\text { Tourino } \\
\text { LFPG, Co- } \\
\text { rrêa-Faria P, } \\
\text { Ferreira RC, } \\
\text { Bendo CB, } \\
\text { Zarzar PM, } \\
\text { Vale MP. }\end{array}$ & 2016 & Brasil & $\begin{array}{l}\text { Estudio } \\
\text { poblacional }\end{array}$ & $\begin{array}{l}8 \text { a } 9 \text { años } y \\
11 \text { meses }\end{array}$ & 1181 niños & $\begin{array}{l}10 \text { escuelas prima- } \\
\text { rias privadas y } 24 \\
\text { públicas en áreas } \\
\text { urbanas y rurales } \\
\text { de Lavras. }\end{array}$ & $\begin{array}{l}\text { Siguiendo las } \\
\text { pautas de la } \\
\text { OMS. }\end{array}$ & $\begin{array}{l}\text { cuestionario } \\
\text { autoadmi- } \\
\text { nistrado } \\
\text { adaptado de } \\
\text { Jälevik et al. }\end{array}$ & $\begin{array}{l}\text { La experiencia de } \\
\text { asma, bronquitis, } \\
\text { neumonía, uso de } \\
\text { antibióticos antes } \\
\text { de los } 4 \text { años se } \\
\text { asoció a HMI. }\end{array}$ & $\begin{array}{l}P=0,001 \\
P=0,006 \\
P=0,029 \\
P=0,025\end{array}$ \\
\hline
\end{tabular}


Tabla 1: Resumen de las características de los estudios seleccionados y sus conclusiones. (Cont.)

\begin{tabular}{|c|c|c|c|c|c|c|c|c|c|c|}
\hline \multirow{2}{*}{ Autor } & \multirow{2}{*}{ Año } & \multirow{2}{*}{ País } & \multirow{2}{*}{$\begin{array}{l}\text { Diseño de } \\
\text { estudio }\end{array}$} & \multirow{2}{*}{$\begin{array}{l}\text { Rango de } \\
\text { edad }\end{array}$} & \multirow{2}{*}{$\begin{array}{l}\text { Tamaño de } \\
\text { muestra }\end{array}$} & \multirow{2}{*}{$\begin{array}{l}\text { Reclutamiento de } \\
\text { pacientes }\end{array}$} & \multicolumn{2}{|c|}{$\begin{array}{l}\text { Instrumentos de } \\
\text { colecta de datos }\end{array}$} & \multirow{2}{*}{ Conclusiones } & \multirow{2}{*}{ Valor $\mathrm{p}$} \\
\hline & & & & & & & $\begin{array}{l}\text { Examen } \\
\text { clínico }\end{array}$ & Cuestionario & & \\
\hline $\begin{array}{l}\text { Mishra A, } \\
\text { Pandey } \\
\text { RK. }^{24}\end{array}$ & 2016 & India & $\begin{array}{l}\text { Estudio epi- } \\
\text { demiológico }\end{array}$ & 8 a 12 años. & 1.369 niños & & $\begin{array}{l}\text { Según los } \\
\text { criterios de la } \\
\text { EAPD. }\end{array}$ & Cuestionario. & $\begin{array}{l}\text { Infección de oídos } \\
\text { y deficiencia de } \\
\text { vitamina A en la } \\
\text { primera infancia se } \\
\text { asoció a HMI. }\end{array}$ & $\begin{array}{l}P=0,03 \\
P=0,04\end{array}$ \\
\hline $\begin{array}{l}\text { Mastora A, } \\
\text { Vadiakas G, } \\
\text { Agouropou- } \\
\text { los A, Garta- } \\
\text { gani-Pana- } \\
\text { giotopoulou } \\
\text { P, Gemou } \\
\text { Engesaeth } \\
\text { V. }^{25}\end{array}$ & 2016 & Grecia & $\begin{array}{l}\text { Retrospecti- } \\
\text { vo de casos } \\
\text { y controles } \\
\text { con niños } \\
\text { griegos }\end{array}$ & 6 a 12 años & $\begin{array}{l}70 \text { casos } 70 \\
\text { controles }\end{array}$ & $\begin{array}{l}\text { Hospital de } \\
\text { Niños 'Aghia So fi a' } \\
\text { Atenas. }\end{array}$ & $\begin{array}{l}\text { Examen } \\
\text { clínico. }\end{array}$ & Cuestionario. & $\begin{array}{l}\text { La rinitis alérgica } \\
\text { y el consumo de } \\
\text { antibióticos en el } \\
\text { período posnatal } \\
\text { parece tener rela- } \\
\text { ción con HMI. }\end{array}$ & $\begin{array}{l}P=0,016 \\
P=0,004\end{array}$ \\
\hline $\begin{array}{l}\text { Rai A, Singh } \\
\text { A, Menon } \\
\text { I, Singh J, } \\
\text { Rai V, Aswal } \\
\text { GS }^{38}\end{array}$ & 2018 & Etiopía & $\begin{array}{l}\text { Estudio } \\
\text { transversal }\end{array}$ & 7 a9 años & 992 niños & $\begin{array}{l}\text { Escuela de Murad- } \\
\text { nagar. }\end{array}$ & $\begin{array}{l}\text { índice } \\
\text { modificado } \\
\text { de (DDE). }\end{array}$ & Cuestionario. & $\begin{array}{l}\text { Período prenatal: } \\
\text { Diabetes gestacio- } \\
\text { nal, presión arterial } \\
\text { hipocalcemia, } \\
\text { deficiencia de } \\
\text { vitamina D. Periodo } \\
\text { perinatal: parto } \\
\text { prematuro y parto } \\
\text { prolongado. Periodo } \\
\text { posnatal: asma, } \\
\text { otitis media, alergia } \\
\text { y medicamento } \\
\text { como la amoxicilina } \\
\text { son los factores de } \\
\text { riesgo que tienen } \\
\text { la mayor fuerza de } \\
\text { asociación con HMI. }\end{array}$ & $P=0,001$ \\
\hline $\begin{array}{l}\text { Dantas-Neta } \\
\text { NB, Soares } \\
\text { Figueiredo } \\
\text { M, Lima } \\
\text { CCB, Bendo } \\
\text { CB, Matos de } \\
\text { Andrade ÉM, } \\
\text { Lima M de } \\
\text { DM, et al. }\end{array}$ & 2018 & Brasil & $\begin{array}{l}\text { Casos y } \\
\text { controles }\end{array}$ & & $\begin{array}{l}186 \text { niños } \\
\text { con HMI } \\
558 \text { niños sin } \\
\text { HMI }\end{array}$ & $\begin{array}{l}\text { Escuelas de Teresi- } \\
\text { na, Brasil. }\end{array}$ & $\begin{array}{l}\text { Según los } \\
\text { criterios de la } \\
\text { EAPD. }\end{array}$ & Cuestionario. & $\begin{array}{l}\text { la presencia de } \\
\text { fiebre durante el } \\
\text { embarazo y en el } \\
\text { periodo posnatal: } \\
\text { uso de antibióticos } \\
\text { se asociaron con } \\
\text { HMI. }\end{array}$ & $\begin{array}{l}P=0,045 \\
P=0,050\end{array}$ \\
\hline $\begin{array}{l}\text { Hernandez } \\
\text { M, Boj JR, } \\
\text { Espasa E. }\end{array}$ & 2018 & España & $\begin{array}{l}\text { Estudio } \\
\text { transversal }\end{array}$ & $\begin{array}{l}6 \text { a } 14 \text { años } \\
11 \text { meses }\end{array}$ & 705 niños & $\begin{array}{l}2 \text { ciudades en la } \\
\text { región central de la } \\
\text { provincia de Barce- } \\
\text { lona, España. }\end{array}$ & $\begin{array}{l}\text { Examen } \\
\text { clínico. }\end{array}$ & Cuestionario. & $\begin{array}{l}\text { HMI fue significati- } \\
\text { vamente más preva- } \\
\text { lente en el periodo } \\
\text { posnatal, entre } \\
\text { aquellos que tenían } \\
\text { dermatitis atópica, } \\
\text { alergias alimenta- } \\
\text { rias, bronquitis / } \\
\text { asma, varicela, otitis } \\
\text { media, neumonía y } \\
\text { síndrome febril. }\end{array}$ & $P=0,001$ \\
\hline $\begin{array}{l}\text { Koruyucu M, } \\
\text { Özel S, Tuna } \\
\text { EB. }{ }^{40}\end{array}$ & 2018 & Estambul & $\begin{array}{l}\text { Estudio } \\
\text { transversal }\end{array}$ & 8 a 11 años & 1511 niños & $\begin{array}{l}\text { Universidad de } \\
\text { Estambul, Facultad } \\
\text { de Odontología. }\end{array}$ & $\begin{array}{l}\text { Examen } \\
\text { clínico. }\end{array}$ & $\begin{array}{l}\text { Cuestionario } \\
\text { a padres. }\end{array}$ & $\begin{array}{l}\text { Complicaciones } \\
\text { durante el embara- } \\
\text { zo de la madre, el } \\
\text { parto prematuro, el } \\
\text { período de lactancia } \\
\text { promedio. En el } \\
\text { periodo posnatal: } \\
\text { la frecuencia } \\
\text { de diarrea, las } \\
\text { enfermedades del } \\
\text { sistema digestivo, } \\
\text { el asma, la fiebre } \\
\text { alta frecuente, la } \\
\text { infección del oído, } \\
\text { la insuficiencia } \\
\text { renal, la rubeola, la } \\
\text { varicela y la paroti- } \\
\text { ditis están asociada } \\
\text { con HMl. }\end{array}$ & $P=0,001$ \\
\hline
\end{tabular}


Tabla 1: Resumen de las características de los estudios seleccionados y sus conclusiones. (Cont.)

\begin{tabular}{|c|c|c|c|c|c|c|c|c|c|c|}
\hline \multirow{2}{*}{ Autor } & \multirow{2}{*}{ Año } & \multirow{2}{*}{ País } & \multirow{2}{*}{$\begin{array}{l}\text { Diseño de } \\
\text { estudio }\end{array}$} & \multirow{2}{*}{$\begin{array}{l}\text { Rango de } \\
\text { edad }\end{array}$} & \multirow{2}{*}{$\begin{array}{l}\text { Tamaño de } \\
\text { muestra }\end{array}$} & \multirow{2}{*}{$\begin{array}{l}\text { Reclutamiento de } \\
\text { pacientes }\end{array}$} & \multicolumn{2}{|c|}{$\begin{array}{l}\text { Instrumentos de colecta de } \\
\text { datos }\end{array}$} & \multirow{2}{*}{ Conclusiones } & \multirow{2}{*}{ Valor $p$} \\
\hline & & & & & & & $\begin{array}{l}\text { Examen } \\
\text { clínico }\end{array}$ & Cuestionario & & \\
\hline $\begin{array}{l}\text { Portella PD, } \\
\text { Fraiz FC, } \\
\text { Soares RC, } \\
\text { Nagata AG, } \\
\text { Tomaz C de } \\
\text { O, Assunção } \\
\text { LR da S. }{ }^{27}\end{array}$ & 2018 & Brasil & $\begin{array}{l}\text { Casos y } \\
\text { controles }\end{array}$ & 6 a 13 años & $\begin{array}{l}31 \text { niños ca- } \\
\text { sos } 62 \text { niños } \\
\text { controles }\end{array}$ & $\begin{array}{l}\text { Clínica de Odon- } \\
\text { tología Pediátrica } \\
\text { de la Universidad } \\
\text { Federal de Paraná, } \\
\text { Brasil }\end{array}$ & $\begin{array}{l}\text { Según los } \\
\text { criterios de la } \\
\text { EAPD }\end{array}$ & $\begin{array}{l}\text { Cuestio- } \\
\text { narios de } \\
\text { percepción } \\
\text { infantil CPQ }\end{array}$ & $\begin{array}{l}\text { En el período } \\
\text { perinatal: Parto } \\
\text { prematuro y el parto } \\
\text { prolongado, En el } \\
\text { período posnatal: } \\
\text { las fiebres recurren- } \\
\text { tes en los primeros } \\
3 \text { años de vida se } \\
\text { asociaron con HMI. }\end{array}$ & $\begin{array}{l}P=0,039 \\
P=0,023 \\
P=0,011\end{array}$ \\
\hline $\begin{array}{l}\text { Flexeder C, } \\
\text { Kabary Has- } \\
\text { san L, Standl } \\
\text { M, Schulz } \\
\text { H, Kühnisch } \\
\text { J. }^{32}\end{array}$ & 2019 & & $\begin{array}{l}\text { Estudios de } \\
\text { cohorte de } \\
\text { nacimiento }\end{array}$ & 10 a 15 años & $\begin{array}{l}730 \text { Adoles- } \\
\text { centes }\end{array}$ & & $\begin{array}{l}\text { Examen clíni- } \\
\text { co ICDAS }\end{array}$ & Cuestionario & $\begin{array}{l}\text { La asociación entre } \\
\text { asma y el uso de } \\
\text { medicación con } \\
\text { HMI. }\end{array}$ & $P=0,043$ \\
\hline $\begin{array}{l}\text { Mejía JD, } \\
\text { Restrepo M, } \\
\text { González S, } \\
\text { Álvarez LG, } \\
\text { Santos-Pinto } \\
\text { L, Escobar } \\
\text { A. }{ }^{29}\end{array}$ & 2019 & $\begin{array}{l}\text { Colom- } \\
\text { bia }\end{array}$ & $\begin{array}{l}\text { Observacio- } \\
\text { nal retros- } \\
\text { pectivo }\end{array}$ & 6 a 15 año & 1.075 niños & $\begin{array}{l}\text { escuelas privadas } \\
\text { o públicas en } \\
\text { la ciudad de } \\
\text { Medellín y fueron } \\
\text { seleccionados por } \\
\text { muestreo aleatorio } \\
\text { estratificado. }\end{array}$ & $\begin{array}{l}\text { Según los } \\
\text { criterios de la } \\
\text { EAPD }\end{array}$ & Cuestionario & $\begin{array}{l}\text { La hipocalcemia en } \\
\text { el primer trimestre } \\
\text { de embarazo, el } \\
\text { parto prematu- } \\
\text { ro, problemas } \\
\text { respiratorios en los } \\
\text { primeros } 3 \text { años de } \\
\text { vida se asociaron } \\
\text { a HMI. }\end{array}$ & $\begin{array}{l}P=0,001 \\
P=0,000 \\
P=0,000\end{array}$ \\
\hline
\end{tabular}

datos (examen clínico, cuestionario), año, diseño de estudio y conclusiones, y en la tabla 2 se consideraron los datos: autor, año, diseño de estudio, factores sistémicos asociados (periodo prenatal, período perinatal, período posnatal) y valor $\mathrm{P}$.
La revisión de los estudios se estableció en tres fases: lectura del título, lectura del resumen y la lectura completa del artículo. Para garantizar que la temática del artículo seleccionada esté relacionada con la temática a estudiar.

Tabla 2: Resumen de los estudios seleccionados presentando sus resultados según periodos del desarrollo afectados por los factores sistémicos estudiados.

\begin{tabular}{|c|c|c|c|c|c|c|}
\hline \multirow{2}{*}{ Autor } & \multirow{2}{*}{ Año } & \multirow{2}{*}{ Diseño } & \multicolumn{3}{|c|}{ Factores sistémicos asociados } & \multirow{2}{*}{ Valor $\mathrm{p}$} \\
\hline & & & Periodo prenatal & Periodo perinatal & Periodo posnatal & \\
\hline $\begin{array}{l}\text { Souza JF, Costa-Silva CM, } \\
\text { Jeremias F, Santos-Pinto } \\
\text { L, Zuanon ACC, Cordeiro } \\
\mathrm{RCL}^{33}\end{array}$ & 2012 & Estudio transversal. & $\begin{array}{l}\text { Problemas médicos duran- } \\
\text { te el embarazo Infecciones } \\
\text { de garganta. Fiebre alta, } \\
\text { uso de Amoxicilina asocia- } \\
\text { da a otros antibióticos. }\end{array}$ & 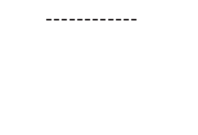 & ----------- & $\begin{array}{l}P=0,01 \\
P=0,02 \\
P=0,04\end{array}$ \\
\hline $\begin{array}{l}\text { Sönmez H, Yildirim G, } \\
\text { Bezgin } \mathrm{T}^{31}\end{array}$ & 2012 & $\begin{array}{l}\text { Estudio epidemio- } \\
\text { lógico. }\end{array}$ & ----------- & Parto prematuro. & $\begin{array}{l}\text { Problemas gastrointestinales, neumo- } \\
\text { nía, fiebre alta frecuente, sarampión y } \\
\text { varicela antes de los } 4 \text { años. }\end{array}$ & $\begin{array}{l}P=0,035 \\
P=0,05\end{array}$ \\
\hline $\begin{array}{l}\text { Durmus B, Abbasoglu Z, } \\
\text { Peker S, Kargul B. }{ }^{35}\end{array}$ & 2013 & Estudio transversal. & Bronquitis. & ---------- & $\begin{array}{l}\text { Consumo prolongado de medica- } \\
\text { mentos. } \\
\text { Asma antes de los } 3 \text { años de vida. } \\
\text { Fiebre. }\end{array}$ & $\begin{array}{l}P=0,029 \\
P=0,006 \\
P=0,05 \\
P=0,05\end{array}$ \\
\hline $\begin{array}{l}\text { Kühnisch J, Mach D, } \\
\text { Thiering E, Brockow I, } \\
\text { Hoffmann U, Neumann } \\
\text { C, et al } \\
28\end{array}$ & 2014 & $\begin{array}{l}\text { Estudio de cohorte de } \\
\text { nacimiento. }\end{array}$ & ----------- & ---------- & $\begin{array}{l}\text { Enfermedades Respiratorias como: } \\
\text { Asma bronquial, bronquitis, neumonía. }\end{array}$ & $P=0,05$ \\
\hline $\begin{array}{l}\text { Ghanim A, Manton D, } \\
\text { Bailey D, Mariño R, } \\
\text { Morgan M. }{ }^{21}\end{array}$ & 2013 & Estudio transversal. & $\begin{array}{l}\text { Hipocalcemia y dificultad } \\
\text { respiratoria. }\end{array}$ & & $\begin{array}{l}\text { Amigdalitis, otitis media, neumonía, } \\
\text { fiebre alta. }\end{array}$ & $\begin{array}{l}P=0,04 \\
P=0,03 \\
P=0,01 \\
P=0,03 \\
P=0,04 \\
P=0,04 \\
P=0,001\end{array}$ \\
\hline
\end{tabular}


Tabla 2: Resumen de los estudios seleccionados presentando sus resultados según periodos del desarrollo afectados por los factores sistémicos estudiados. (Cont.)

\begin{tabular}{|c|c|c|c|c|c|c|}
\hline \multirow{2}{*}{ Autor } & \multirow{2}{*}{ Año } & \multirow{2}{*}{ Diseño } & \multicolumn{3}{|c|}{ Factores sistémicos asociados } & \multirow{2}{*}{ Valor $\mathrm{p}$} \\
\hline & & & Periodo prenatal & Periodo perinatal & Periodo posnatal & \\
\hline $\begin{array}{l}\text { Allazzam SM, Alaki SM, } \\
\text { Sadek A, Meligy E }\end{array}$ & 2014 & Estudio transversal. & ---------- & ----------- & $\begin{array}{l}\text { Amigdalitis, adenoiditis, otitis media. } \\
\text { Ingesta frecuente de antibióticos. } \\
\text { Asma. } \\
\text { Fiebre. }\end{array}$ & $\begin{array}{l}P=0,001 \\
P=0,001 \\
P=0,001 \\
P=0.014\end{array}$ \\
\hline $\begin{array}{l}\text { Pitiphat W, Luangchai- } \\
\text { chaweng S, Pungchan- } \\
\text { chaikul P, Angwaravong } \\
\text { O, Chansamak N. }\end{array}$ & 2014 & Estudio transversal. & ----------- & $\begin{array}{l}\text { Cesárea, el parto } \\
\text { vaginal complicado. }\end{array}$ & $\begin{array}{l}\text { Asma. } \\
\text { Uso de antibióticos antes de los } 3 \\
\text { años de vida. }\end{array}$ & $\begin{array}{l}P<0,001 \\
P=0,001 \\
P=0,01\end{array}$ \\
\hline $\begin{array}{l}\text { Tourino LFPG, Corrêa-Fa- } \\
\text { ria P, Ferreira RC, Bendo } \\
\text { CB, Zarzar PM, Vale } \\
\text { MP. }^{30}\end{array}$ & 2016 & Estudio poblacional. & ----------- & ----------- & $\begin{array}{l}\text { Asma / bronquitis antes de } 4 \text { años. } \\
\text { Neumonía, fiebre. } \\
\text { Uso de antibióticos. }\end{array}$ & $\begin{array}{l}P=0,001 \\
P=0,006 \\
P=0,029 \\
P=0,025\end{array}$ \\
\hline Mishra A, Pandey RK. ${ }^{24}$ & 2016 & $\begin{array}{l}\text { Estudio epidemio- } \\
\text { lógico. }\end{array}$ & ----------- & ----------- & $\begin{array}{l}\text { Deficiencia de vitamina A y la infec- } \\
\text { ción del oído en la primera infancia. }\end{array}$ & $\begin{array}{l}P=0,03 \\
P=0,04\end{array}$ \\
\hline $\begin{array}{l}\text { Wuollet E, Laisi S, Salmela } \\
\text { E, Ess A, Alaluusua S. }{ }^{34}\end{array}$ & 2016 & $\begin{array}{l}\text { Estudio de cohorte } \\
\text { retrospectivo. }\end{array}$ & ---------- & & $\begin{array}{l}\text { Otitis media aguda durante el primer } \\
\text { año de vida. Los niños que habían re- } \\
\text { cibido al menos un ciclo de penicilina } \\
\text { durante el primer año de vida. }\end{array}$ & $\begin{array}{l}P=0,035 \\
P=0,019\end{array}$ \\
\hline $\begin{array}{l}\text { Rai A, Singh A, Menon } \\
\text { I, Singh J, Rai V, Aswal } \\
\text { GS }^{38}\end{array}$ & 2018 & Estudio transversal. & $\begin{array}{l}\text { Diabetes gestacional, pre- } \\
\text { sión arterial, hipocalcemia, } \\
\text { deficiencia de vitamina } \mathrm{D} \text {. }\end{array}$ & Parto prematuro. & $\begin{array}{l}\text { Enfermedades como asma, otitis } \\
\text { media, alergia y medicamento como la } \\
\text { amoxicilina. }\end{array}$ & $P=0,001$ \\
\hline $\begin{array}{l}\text { Dantas-Neta NB, Soares } \\
\text { Figueiredo M, Lima CCB, } \\
\text { Bendo CB, Matos de } \\
\text { Andrade ÉM, Lima M de } \\
\text { DM, et al. }{ }^{26}\end{array}$ & 2018 & Casos y controles. & $\begin{array}{l}\text { Fiebre durante el em- } \\
\text { barazo. }\end{array}$ & ---------- & Uso de antibióticos. & $\begin{array}{l}P=0,045 \\
P=0,050\end{array}$ \\
\hline $\begin{array}{l}\text { Hernandez M, Boj JR, } \\
\text { Espasa E.. }{ }^{39}\end{array}$ & 2018 & Estudio transversal. & & & $\begin{array}{l}\text { Dermatitis atópica, alergias alimenta- } \\
\text { rias, bronquitis / asma, varicela, otitis } \\
\text { media, neumonía y síndrome febril. }\end{array}$ & $P=0,001$ \\
\hline $\begin{array}{l}\text { Koruyucu M, Özel S, Tuna } \\
\text { EB. }{ }^{40}\end{array}$ & 2018 & Estudio transversal. & $\begin{array}{l}\text { Complicaciones en el } \\
\text { embrazo. }\end{array}$ & Parto prematuro. & $\begin{array}{l}\text { Frecuencia de diarrea, las enfermeda- } \\
\text { des del sistema digestivo, el asma, la } \\
\text { fiebre alta frecuente, la infección del } \\
\text { oído, la insuficiencia renal, la rubeola, } \\
\text { la varicela y la parotitis. }\end{array}$ & $P=0,001$ \\
\hline $\begin{array}{l}\text { Portella PD, Fraiz FC, } \\
\text { Soares RC, Nagata AG, } \\
\text { Tomaz C de O, Assunção } \\
\text { LR da S. }{ }^{27}\end{array}$ & 2018 & Casos y controles. & & $\begin{array}{l}\text { Parto prematuro. } \\
\text { Parto prolongado. }\end{array}$ & Fiebres recurrentes. & $\begin{array}{l}P=0,039 \\
P=0,023 \\
P=0,011\end{array}$ \\
\hline $\begin{array}{l}\text { Flexeder C, Kabary Has- } \\
\text { san L, Standl M, Schulz H, } \\
\text { Kühnisch J. }\end{array}$ & 2019 & $\begin{array}{l}\text { Estudios de cohorte } \\
\text { de nacimiento. }\end{array}$ & ---------- & ---------- & Asma y medicación. & $P=0,043$ \\
\hline $\begin{array}{l}\text { Mejía JD, Restrepo M, } \\
\text { González S, Álvarez LG, } \\
\text { Santos-Pinto L, Escobar } \\
\text { A. }{ }^{29}\end{array}$ & 2019 & $\begin{array}{l}\text { Observacional retros- } \\
\text { pectivo. }\end{array}$ & Hipocalcemia. & Parto prematuro. & Problemas respiratorios. & $\begin{array}{l}P=0,001 \\
P=0,000 \\
P=0,000\end{array}$ \\
\hline
\end{tabular}

\section{Resultados}

\section{Selección del estudio}

En la búsqueda inicial se recopilaron 115 artículos de los cuales:

10 base de datos Pubmed, 45 Hinari, 6 biblioteca Cochrane y 54 en diferentes revistas científicas indexadas. Antes de iniciar las fases de selección se eliminaron 11 artículos que estaban duplicados, es decir, se encontraban en dos bases de datos, 40 artículos fueron excluidos porque no existía relación entre las implicaciones sistémicas con $\mathrm{HMI}$ y 16 revisiones sistemáticas y 
metaanálisis por no considerarse estudios primarios.

De los 115 artículos seleccionados inicialmente, 48 artículos fueron elegibles en la primera fase de lectura del título, excluyéndose 11 artículos por encontrarse asociados a otros factores etiológicos. Siendo 37 artículos elegibles para iniciar con la segunda fase de lectura del resumen donde 16 fueron excluidos por no existir ninguna asociación con HMI.
Así, 22 artículos fueron elegibles para iniciar con la tercera fase de lectura completa del artículo, en la cual, 3 artículos no mostraron asociación entre ninguna de las implicaciones sistémicas, quedando finalmente 18 estudios como elegibles para ser incluidos en la presente revisión.

Dicho proceso, de selección y eliminación de artículos se grafica a través de un flujograma (Figura 1)

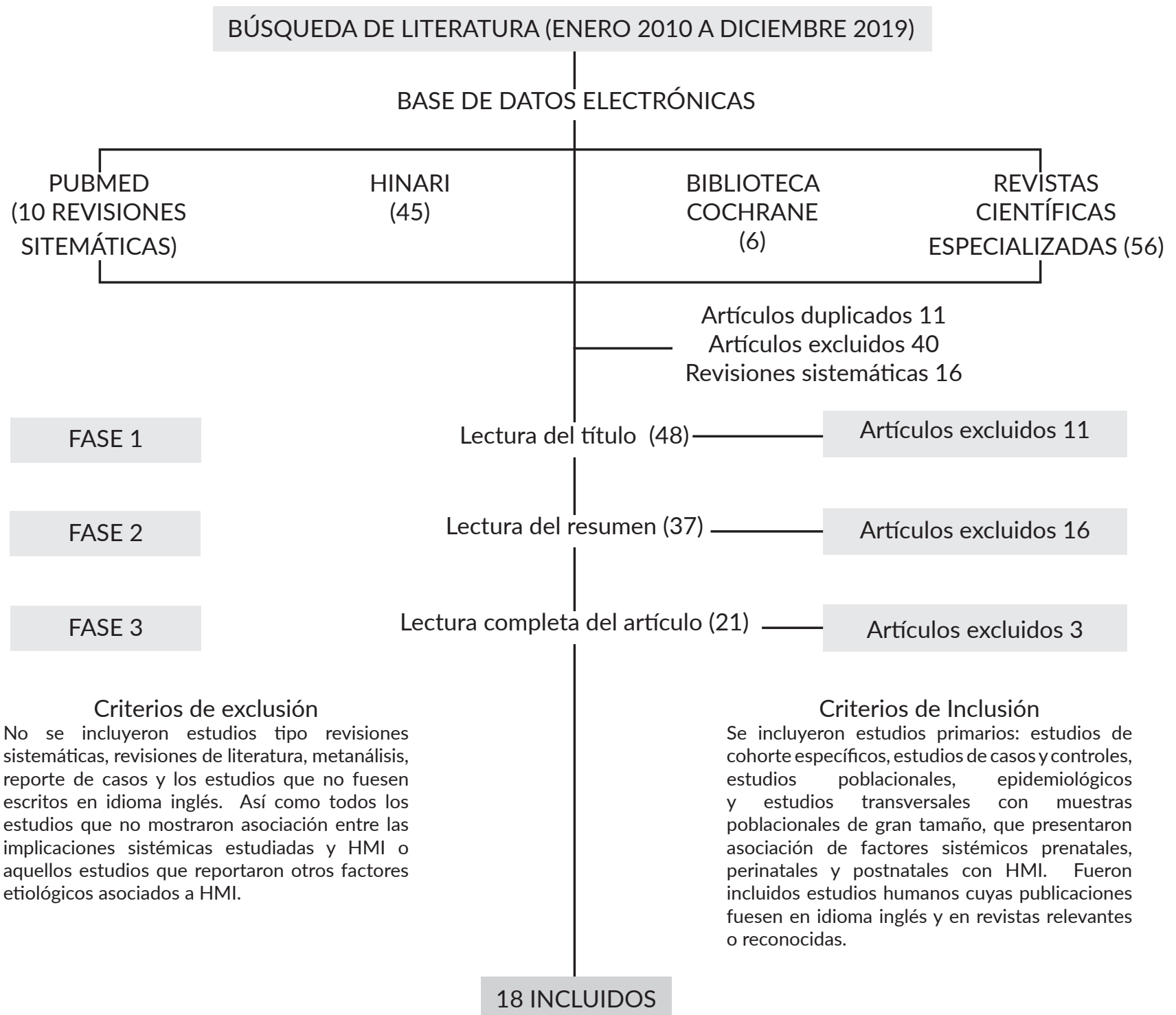

Figura 1: Flujograma proceso de búsqueda revisión de literatura 


\section{Características de artículos incluidos}

De los estudios seleccionados para esta revisión de literatura ocho fueron estudios transversales, ${ }^{21,33,35,36-40}$ tres estudios de casos y controles,,$^{25,26,27}$ un estudio de cohorte prospectivo, ${ }^{17}$ tres estudios de cohorte retrospectivo, ${ }^{34,28,25}$ un estudio observacional retrospectivo, ${ }^{29}$ un estudio poblacional ${ }^{30}$ y dos estudios epidemiológicos. ${ }^{31,41}$ El tamaño de la muestra varió entre 90 a 6,690 niños, con edades comprendidas entre los 9 a 14 años.

\section{Criterios de elegibilidad}

Se incluyeron estudios primarios: estudios de cohorte específicos, estudios de casos y controles, estudios poblacionales, epidemiológicos y estudios transversales con muestras poblacionales de gran tamaño, que presentaron asociación de factores sistémicos prenatales, perinatales y postnatales con HMI.

Fueron incluidos estudios humanos cuyas publicaciones fuesen en idioma inglés y en revistas relevantes o reconocidas.

\section{Criterios de exclusión}

Fueron excluidas las publicaciones duplicadas, informes de casos, revisiones de literatura, revisiones sistemáticas, metanálisis por no considerarse estudios primarios y artículos en otro idioma que no fuese inglés. Fueron excluidos también los estudios que no mostraron asociación entre las implicaciones sistémicas y HMI y aquellos estudios que reportaron asociación con otros factores etiológicos como por ejemplo la genética o factores ambientales.
La relevancia de los resultados encontrados en la presente revisión de literatura radica en la importancia de presentar de forma sistematizada las implicaciones sistémicas asociadas a $\mathrm{HMI}$ consideradas hasta la fecha dentro de la mejor evidencia científica disponible, facilitando una postura más acertada por parte del clínico dentista que le permita enfocarse en educación y prevención al momento del abordaje clínico de los pacientes que presenten este tipo de alteración del esmalte dentario.

\section{Discusión}

A pesar de los múltiples esfuerzos realizados por investigadores, la etiología de la HMI en la actualidad aún se mantiene como incierta y sigue sin definirse en su totalidad, sin embargo, se contempla como una alteración de origen multifactorial y compleja. ${ }^{42}$ Los trabajos realizados hasta la fecha permiten estudiar los posibles factores sistémicos asociados con HMI que se presentan en periodos específicos y críticos del desarrollo infantil considerados desde el último trimestre de embarazo hasta aproximadamente los primeros tres años de vida, periodo que coincide con el delicado y complejo proceso de amelogénesis. ${ }^{23}$

\section{Período prenatal}

El embarazo es un periodo de muchos cambios en el organismo de la mujer que carga en su vientre un nuevo ser quien se encuentra en constante desarrollo y crecimiento desde el momento mismo de la concepción y cualquier alteración que afecte la salud de la madre afectará directamente el buen desarrollo del feto, incluyendo el proceso mismo de amelogénesis. 
Souza JF et al. en $2013,{ }^{6}$ al igual que otros autores $^{29,38,40,33}$ sugieren que existe un vínculo entre la $\mathrm{HMI}$ y los problemas de salud o complicaciones reportados por la madre en el período del embarazo. Entre los factores sistémicos asociados con HMI reportados por las gestantes pueden resaltarse complicaciones como: fiebres altas, hipertensión arterial, diabetes gestacional, procesos infecciosos, uso frecuente de medicamentos (antibióticos) y desnutrición. ${ }^{26,29,34,38,43}$

Amoxicilina es el medicamento más asociado con la presencia de $\mathrm{HMI}$, sin embargo este dato aún es controversial porque no se sabe con certeza absoluta si el propio medicamento altera el proceso de amelogénesis o si es la combinación de este y las condiciones sistémicas del paciente en ese momento específico, es decir; los antibióticos son prescritos cuando se presentan procesos infecciosos que vienen acompañados de un cuadro clínico de fiebre cuyo aumento de temperatura sí afecta el proceso de amelogénesis., 4

En relación al uso de medicamentos durante el embarazo, Serna C et al. en $2016,{ }^{44}$ determinaron que la evidencia disponible sugiere que no se puede decir inequívocamente que ningún medicamento durante el embarazo pueda ser considerado como predictor de HMI. por lo que una anamnesis minuciosa en el control prenatal o durante la primera consulta odontológica del bebé puede arrojar datos que nos lleven a orientar y alertar a los responsables y también integrar esta información para asociarla con las posibles causas del aparecimiento de estos defectos al momento de la erupción dentaria. ${ }^{45}$
Entre otros factores asociados, Souza JF, et al. en $2016,{ }^{33}$ sugiere un vínculo entre $\mathrm{HMI}$ y problemas de salud durante el embarazo, así como factores ambientales. Coincidiendo con un estudio realizado por Fatturi AL et al. en 2019, ${ }^{42}$ donde se establece que el período prenatal y el estrés psicológico durante la gestación se asoció con HMI, este metanálisis mostró que los niños cuyas madres tuvieron problemas de salud durante la gestación presentaron un $40 \%$ más de probabilidades de $\mathrm{HMI}$ que los hijos de madres que no tuvieron problemas en la gestación. La revisión se llevó a cabo en Brasil y consideró estudios de países como Corea del sur e Irak que en ese momento se encontraban en constante tensión por tiempos de guerra, mostrando que el estrés y la ansiedad son condiciones psicológicas asociadas con diversos problemas de salud. ${ }^{42}$

\section{Periodo perinatal}

Durante el proceso del parto pueden también presentarse complicaciones que han sido asociadas con la presencia de $\mathrm{HMI}$, entre ellas se mencionan: partos por cesárea, parto vaginal complicado, partos prolongados, bajo peso al nacer y prematuridad. $27,37,40$

En relación a la prematuridad según la OMS, se estima que cada año nacen unos 15 millones de niños prematuros y esta cifra está aumentando. ${ }^{46}$ Un estudio epidemiológico realizado por Sönmez $\mathrm{HH}$ et al. en 2013, ${ }^{31}$ encontró que la HMI estaba asociada a la prematuridad en un $7 \%$. Por lo tanto, reconocer esta realidad creciente, alerta al clínico dentista para acompañar de cerca este tipo de paciente y su núcleo familiar. 
Otros estudios al igual que Sönmez, concordaron con este resultado evidenciando que el parto prematuro se encontraba significativamente asociado a HMI. ${ }^{40}$

Una reciente revisión sistemática confirma estos resultados donde se determinó que el nacimiento prematuro promovió la prevalencia de HMI (OR 1/4 1,57, IC 95\%: 1,07- 2,31), asociándolo también con bajo peso al nacer, condición que puede estar ligada a la prematuridad del recién nacido. Los recién nacidos de bajo peso al nacer tenían aproximadamente tres veces más probabilidades de sufrir HMI (OR $1 / 43,25$, IC 95\%: 2,28 - 4,62) 47 datos que coinciden con otros estudios. ${ }^{48,29}$

Contrario a estos resultados se encontró el estudio de Fatturi AL et al. en 2019,42 en una revisión sistemática estableciendo que no existe una relación concreta entre la prematuridad, la lactancia materna y el bajo peso al nacer con HMl. sin embargo, estos datos deben ser analizados con cautela. ${ }^{42}$

\section{Periodo postnatal}

El periodo de los primeros tres años de vida del niño es un periodo importante en su desarrollo integral y existe el riesgo de enfermedades frecuentes. Kühnisch J et al. en $2014,{ }^{28}$ en un estudio de cohorte de nacimiento confirmó que las enfermedades respiratorias tempranas parecen estar directa e indirectamente relacionadas con HMI. Se han reportado como problemas respiratorios: asma, neumonía, rinitis, infecciones adenoideas que se ven asociadas con la presencia de fiebre como factores relacionados con $\mathrm{HMI}^{25,27,30,32,34-}$ $36,38,40,42$
Tourino LFPG et al. en 2016, ${ }^{30}$ en un estudio de base poblacional en una muestra de 1181 niños, observaron la asociación de asma y $\mathrm{HMI}$ en los primeros 4 años de vida del niño, lo que requiere y orienta a realizar un tratamiento con un enfoque multidisciplinario. ${ }^{30}$

Flexeder $C$ et al. en 2020, ${ }^{32}$ en un estudio epidemiológico de cohorte de nacimiento puntualizan que la asociación entre asma y medicación con $\mathrm{HMI}$ requiere de una mayor confirmación. Sin embargo, Lima LRS et al. en $2020,{ }^{48}$ en una revisión sistemática reportaron que el asma en el primer año de vida está asociado a HMI.

Pitiphat $\mathrm{W}$ et al. en $2014,{ }^{37}$ realizaron un estudio transversal en Tailandia con 420 niños en las que se encontró que la mala salud durante los primeros 3 años de vida son factores de riesgos independientes para HMI. Lo que refuerza lo encontrado por Mishra A et al. en 2016, ${ }^{24}$ que identificaron en sus estudios independientes que las infecciones postnatales juegan un papel importante en la hipomineralización de molares e incisivos.

Otras enfermedades como problemas gastrointestinales, sarampión, varicela, procesos infecciosos como otitis media, dermatitis atópicas, alergias alimentarias, insuficiencia renal, parotiditis y el consumo constante de medicamentos, entre ellos antibióticos en la infancia temprana fueron asociados con la presencia de HMI. 4,17,29,3436,40

Además, de los posibles factores sistémicos involucrados, Vieira en 2016, ${ }^{10}$ y Bussaneli en $2019,{ }^{49}$ plantean la posibilidad de un papel genético en la etiología de HMI, 
lo que indica que una variación genética puede interactuar con factores sistémicos que conducen a $\mathrm{HMI},{ }^{10,49}$ sosteniendo de esta forma que el origen o la etiología para esta condición clínica conocida como HMI es multifactorial y necesita de más estudios a futuro.

\section{Conclusiones}

Es evidente la alta susceptibilidad de la gestante (periodo prenatal) y de los niños en sus primeros años de vida (periodos peri y posnatal) para la adquisición de enfermedades sistémicas que afecten el normal desarrollo de su organismo que los predispone al desarrollo de HMI, considerando que existen otros factores asociados a su etiología como el genético y ambiental.

Existe una amplia evidencia significativa entre las implicaciones sistémicas prenatales, perinatales y posnatales que se asocian a HMI, sin embargo, no son del todo concluyentes ya que la mayoría de los estudios son estudios transversales, por lo tanto, se considera necesario la realización de más estudios tipo longitudinal prospectivo. Sin embargo, en base a las evidencias disponibles, la anamnesis minuciosa y detallada debe ser siempre considerada como una herramienta valiosa a la hora de la atención Odontológica ya que dicha información puede ser confrontada con los hallazgos clínicos y dar una pauta del momento aproximado donde esas alteraciones afectaron el proceso normal de amelogénesis.
El Odontopediatra juega un papel importante en el diagnóstico y correcto tratamiento de este tipo de alteraciones en los pacientes pediátricos, debido a que es uno de los tantos profesionales de la salud que tiene acceso a la madre en su fase prenatal como al círculo familiar en el periodo posnatal, logrando impactar de forma positiva en la calidad de vida de los niños afectados por HMI.

\section{Recomendaciones}

- Es importante conocer a profundidad sobre la etiología de HMI, específicamente los factores sistémicos asociados para poder hacer el correcto abordaje de estos pacientes y a partir de este conocimiento elaborar guía diagnóstica que nos faciliten la anamnesis, recolección e interpretación de datos que nos conduzcan al entendimiento de las características clínicas y al planeamiento adecuado del tratamiento oportuno.

- Promover la importancia de la Odontología prenatal y el trabajo del odontólogo como parte de un equipo transdisciplinar que orienta y empodera a la gestante para que tenga la mejor calidad de vida, ya que alteraciones en esta fase como la desnutrición, entre otras, se refleja en las condiciones de vida del neonato: prematuridad y bajo peso al nacer que se asocian a la presencia de HMI.

\section{Referencias bibliográficas}

1. Seow WK. Clinical diagnosis of enamel defects: Pitfalls and practical guidelines. International Dental Journal. 1997;47(3):173-82.

2. Alaluusua S. Aetiology of Molar-Incisor Hypomineralisation: A systematic review. European archives of paediatric dentistry : official journal of the European Academy of Paediatric Dentistry. 2010;11(2):53-8. 
3. Muñoz AJ, Meléndez JD, González CV, Sánchez CZ. Frecuencia y Severidad de la Hipomineralización Molar Incisal en Pacientes Atendidos en las Clínicas Odontológicas de la Universidad de La Frontera, Chile. Int J Odontosmat. 2011;5(2):133-40.

4. Wuollet E, Laisi S, Salmela E, Ess A, Alaluusua S. Background factors of molar-incisor hypomineralization in a group of Finnish children. Vol. 72, Acta odontologica Scandinavica. 2014;(1).963-9.

5. Dąbrowski P, Kulus M, Grzelak J, Radzikowska M, Oziembłowski M, Domagała Z, et al. Assessing weaning stress - relations between enamel hypoplasia, $\delta 180$ and $\delta 13 C$ values in human teeth obtained from early modern cemeteries in Wroclaw, Poland. Annals of anatomy = Anatomischer Anzeiger : official organ of the Anatomische Gesellschaft. 2020;2(32):1-13

6. Souza JF, Jeremias F, Costa-Silva CM, Santos-Pinto L, Zuanon ACC, Cordeiro RCL. Aetiology of molarincisor hypomineralisation (MIH) in Brazilian children. European Archives of Paediatric Dentistry. 2013;14(4):233-8.

7. Weerheijm KL, Jälevik B, Alaluusua S. Molar-Incisor Hypomineralisation. Caries Research. 2001;35(5):390-1.

8. Weerheijm KL, Mejàre I. Molar incisor hypomineralization: A questionnaire inventory of its occurrence in member countries of the European Academy of Paediatric Dentistry (EAPD). International Journal of Paediatric Dentistry. 2003;13(6):411-6.

9. Jeremias F, Pierri RAG, Souza JF, Fragelli CMB, Restrepo M, Finoti LS, et al. Family-Based Genetic Association for Molar-Incisor Hypomineralization. Caries Research. 2016;50(3):310-8.

10. Vieira AR, Kup E. On the Etiology of Molar-Incisor Hypomineralization. Caries Research. 2016;50(2):166-9.

11. Elfrink MEC, GhanimA, Manton DJ, Weerheijm KL. Standardised studies on Molar Incisor Hypomineralisation (MIH) and Hypomineralised Second Primary Molars (HSPM): a need. European Archives of Paediatric Dentistry. 2015;16(3):247-55.

12. Reyes MRT, Fatturi AL, Menezes JVNB, Fraiz FC, da Silva Assunção LR, de Souza JF. Demarcated opacity in primary teeth increases the prevalence of molar incisor hypomineralization. Brazilian Oral Research. 2019;33:1-9.

13. Garot E, Denis A, Delbos Y, Manton D, Silva M, Rouas P. Are hypomineralised lesions on second primary molars (HSPM) a predictive sign of molar incisor hypomineralisation (MIH)? A systematic review and a meta-analysis. Journal of Dentistry. 2018;(72):8-13.

14. Da Silva Figueiredo Sé MJ, Ribeiro APD, Dos Santos-Pinto LAM, De Cassia Loiola Cordeiro R, Cabral RN, Leal SC. Are hypomineralized primary molars and canines associated with molar-incisor hypomineralization? Pediatric Dentistry. 2017;39(7):445-9.

15. Mittal R, Chandak S, Chandwani M, Singh P, Pimpale J. Assessment of association between molar incisor hypomineralization and hypomineralized second primary molar. Journal of International Society of Preventive and Community Dentistry. 2016;6(1):34-9.

16. Quintero Y, Restrepo M, Saldarriaga JA, Saldarriaga A, Santos-Pinto L. Treatment options for deciduous molar hypomineralization: A report of three cases. Dental Update. 2019;46(6):546-553.

17. Elfrink MEC, Moll HA, Kiefte-de Jong JC, Jaddoe VWV, Hofman A, Ten Cate JM, et al. Pre- and postnatal determinants of deciduous molar hypomineralisation in 6-year-old children. The generation R study. PLoS ONE. 2014;9(7):1-8.

18. Zhao D, Dong B, Yu D, Ren Q, Sun Y. The prevalence of molar incisor hypomineralization: evidence from 70 studies. International Journal of Paediatric Dentistry. 2018;28(2):170-9.

19. Sidhu N, Wang Y, Barrett E, Casas M. Prevalence and presentation patterns of enamel hypomineralisation (MIH and HSPM) among paediatric hospital dental patients in Toronto, Canada: a cross-sectional study. European Archives of Paediatric Dentistry. 2020;21(2):263-70.

20. Salgado Peralvo À, Peralvo Torres V, Torres A, Torres Mateos M, Ribas Pérez D, Castaño Séiquer C. Prevalencia del síndrome de hipomineralización incisivo-molar: revisión de la literatura. Odontología pediátrica. 2016;24(2):134-48.

21. Ghanim A, Manton D, Bailey D, Mariño R, Morgan M. Risk factors in the occurrence of molar-incisor hypomineralization amongst a group of Iraqi children. International Journal of Paediatric Dentistry. 2013;23(3):197-206.

22. Subramaniam P, Gupta T, Sharma A. Prevalence of molar incisor hypomineralization in 7-9-year-old children of Bengaluru City, India. Contemporary Clinical Dentistry. 2016; 1(6): 11-5.

23. Crombie F, Manton D, Kilpatrick N. Aetiology of molar-incisor hypomineralization: A critical review. International Journal of Paediatric Dentistry. 2009;19(2):73-83.

24. Mishra A, Pandey RK. Molar Incisor Hypomineralization: An Epidemiological Study with Prevalence and Etiological Factors in Indian Pediatric Population. International Journal of Clinical Pediatric Dentistry. 2016;9(2):167-71. 
25. Mastora A, Vadiakas G, Agouropoulos A, Gartagani-Panagiotopoulou P, Gemou Engesaeth V. Developmental defects of enamel in first permanent molars associated with use of asthma drugs in preschool aged children: A retrospective case-control study. European Archives of Paediatric Dentistry. 2017;18(2):105-11.

26. Dantas-Neta NB, Soares Figueiredo M, Lima CCB, Bendo CB, Matos de Andrade ÉM, Lima M de DM, et al. Factors associated with molar-incisor hypomineralisation in schoolchildren aged 8-10 years: a casecontrol study. International Journal of Paediatric Dentistry. 2018;28(6):570-7.

27. Portella PD, Fraiz FC, Soares RC, Nagata AG, Tomaz C de O, Assunção LR da S. Molar-incisor hypomineralization and associated factors: A case-control study. Pesquisa Brasileira em Odontopediatria e Clinica Integrada. 2018,1(18): 1-10.

28. Kühnisch J, Mach D, Thiering E, Brockow I, Hoffmann U, Neumann C, et al. Respiratory diseases are associated with molar-incisor hypomineralizations. Swiss dental journal. 2014;124(3):286-93.

29. Mejía JD, Restrepo M, González S, Álvarez LG, Santos-Pinto L, Escobar A. Molar incisor hypomineralization in Colombia: Prevalence, severity and associated risk factors. Journal of Clinical Pediatric Dentistry. 2019; 1(43):185-9.

30. Tourino LFPG, Corrêa-Faria P, Ferreira RC, Bendo CB, Zarzar PM, Vale MP. Association between molar incisor hypomineralization in schoolchildren and both prenatal and postnatal factors: A population-based study. PLoS ONE. 2016;11(6):1-12.

31. Sönmez H, Yildirim G, Bezgin T. Putative factors associated with molar incisor hypomineralisation: An epidemiological study. European Archives of Paediatric Dentistry. 2013;14(6):375-80.

32. Flexeder C, Kabary Hassan L, Standl M, Schulz H, Kühnisch J. Is There an Association between Asthma and Dental Caries and Molar Incisor Hypomineralisation? Caries Research. 2020;54(1):87-95.

33. Souza JF, Costa-Silva CM, Jeremias F, Santos-Pinto L, Zuanon ACC, Cordeiro RCL. Molar incisor hypomineralisation: Possible aetiological factors in children from urban and rural areas. European Archives of Paediatric Dentistry. 2012;13(4):164-70.

34. Wuollet E, Laisi S, Salmela E, Ess A, Alaluusua S. Molar-incisor hypomineralization and the association with childhood illnesses and antibiotics in a group of Finnish children. Acta Odontologica Scandinavica. 2016;74(5):416-22.

35. Durmus B, Abbasoglu Z, Peker S, Kargul B. Possible Medical Aetiological Factors and Characteristics of Molar Incisor Hypomineralisation in a Group of Turkish Children. Acta Stomatologica Croatica. 2013;47(4):297305.

36. Allazzam SM, Alaki SM, El Meligy OAS. Molar incisor hypomineralization, prevalence, and etiology. International Journal of Dentistry. 2014;2014.

37. Pitiphat W, Luangchaichaweng S, Pungchanchaikul P, Angwaravong O, Chansamak N. Factors associated with molar incisor hypomineralization in Thai children. European Journal of Oral Sciences. 2014;122(4):26570.

38. Rai A, Singh A, Menon I, Singh J, Rai V, Aswal GS. Molar Incisor Hypomineralization: Prevalence and Risk Factors Among 7-9 Years Old School Children in Muradnagar, Ghaziabad. The Open Dentistry Journal. 2018;12(1):714-22.

39. Hernandez M, Boj JR, Espasa E. Do we really know the prevalence of MIH? Journal of Clinical Pediatric Dentistry. 2016;40(4):259-63.

40. Koruyucu M, Özel S, Tuna EB. Prevalence and etiology of molar-incisor hypomineralization (MIH) in the city of Istanbul. Journal of Dental Sciences. 2018;13(4):318-28.

41. Sarkar P, Dasar P, Nagarajappa S, Mishra P, Kumar S, Balsaraf S, et al. Impact of dental neglect scale on oral health status among different professionals in Indore city-A cross- sectional study. Journal of Clinical and Diagnostic Research. 2015;9(10):ZC67-70.

42. Fatturi AL, Wambier LM, Chibinski AC, Assunção LR da S, Brancher JA, Reis A, et al. A systematic review and meta-analysis of systemic exposure associated with molar incisor hypomineralization. Community Dentistry and Oral Epidemiology. 2019;47(5):407-15.

43. Da Costa Silva CM, Jeremias F, Feltrin de Souza J, Cilense Zuanon ÂC, Loiola Cordeiro R de C, Dos SantosPinto L. Hipominerazación de incisivos y molares: aspectos clínicos de la severidad. Acta odontol venez. 2010;1-9.

44. Serna C, Vicente A, Finke C, Ortiz AJ. Drugs related to the etiology of molar incisor hypomineralization A systematic review., Journal of the American Dental Association. Elsevier Inc. 2016, 1(147):120-30.

45. Serna Muñoz C, Ortiz Ruiz AJ, Pérez Silva A, Bravo-González LA, Vicente A. Second primary molar hypomineralisation and drugs used during pregnancy and infancy. A systematic review. Clinical Oral Investigations. 2020;24(3):1287-97.

46. OMS. Nacimientos prematuros Visión general. Organización Mundial de la Salud. 2019;1-6. 
47. Wu X, Wang J, Li Y heng, Yang Z yan, Zhou Z. Association of molar incisor hypomineralization with premature birth or low birth weight: systematic review and meta-analysis. Journal of Maternal-Fetal and Neonatal Medicine. 2020;33(10):1700-8.

48. Lima LRS, Pereira AS, de Moura MS, Lima CCB, Paiva SM, Moura L de FA de D, et al. Pre-term birth and asthma is associated with hypomineralized second primary molars in pre-schoolers: A population-based study. Vol. 30, International Journal of Paediatric Dentistry. 2020. p. 193-201.

49. Bussaneli DG, Restrepo M, Fragelli CMB, Santos-Pinto L, Jeremias F, Cordeiro RDCL, et al. Genes Regulating Immune Response and Amelogenesis Interact in Increasing the Susceptibility to Molar-Incisor Hypomineralization. Caries Research. 2019;53(2):217-27.

Recibido: $15 / 10 / 2020$

Aceptado: 17/04/2021

Correspondencia: Nely Arely Garcia de Batres, correo: nely.garcia@ues.edu.sv 